\title{
Hepatic Arterial Infusion Chemotherapy: A Potential Therapeutic Option for Hepatocellular Carcinoma with Portal Vein Tumor Thrombus
}

\author{
Michihisa Moriguchi ${ }^{a}$ Takeshi Aramaki ${ }^{b}$ Toshihiro Tanakac ${ }^{c}$ Yoshito Itoh $^{a}$ \\ a Department of Molecular Gastroenterology and Hepatology, Kyoto Prefectural \\ University of Medicine, Kyoto, Japan; ${ }^{b}$ Interventional Radiology, Shizuoka Kenritsu Shizuoka \\ Gan Center, Shizuoka, Japan; ' Department of Radiology, Nara Medical University, \\ Kashihara, Japan
}

Dear Editor,

Portal vein tumor thrombus (PVTT) is a relatively common form of tumor development during the course of hepatocellular carcinoma (HCC). When advancement into the first branch or main trunk of the portal vein occurs, treatment options are limited, and prognosis is extremely poor.

For patients with Child-Pugh class A advanced HCC with PVTT, systemic chemotherapy is recommended according to the Barcelona-Clinic Liver Cancer (BCLC) staging and treatment strategy. However, the efficacy of currently available systemic therapy is limited, and it is difficult to determine whether the results are satisfactory.

Regression of PVTT is considered important for treatment continuation and prognosis improvement because patients with HCC with advanced PVTT have a decreased liver function and symptoms of hepatic failure, which are not only associated with background liver disease but also with decreased portal blood flow/portal hypertension related to portal vein tumor invasion and decreased performance status. However, currently available systemic chemotherapeutic agents are used with the aim of disease control; therefore, tumor shrinkage or regression of PVTT cannot be expected. This is one factor explaining the limitations of therapeutic effects.

On the other hand, hepatic arterial infusion chemotherapy (HAIC) is sometimes performed for advanced HCC. HAIC is administered via two methods: one-shot HAIC, in which hepatic arterial infusion is repeatedly performed by inserting a catheter each time, and HAIC with a reservoir (implantable port) catheter system, in which a reservoir is placed. Although several regimens for HAIC exist, previous studies have shown that reservoir HAIC may result in a better response [1].

In our retrospective study, HAIC with the reservoir catheter system showed a relatively high response rate and improved prognoses in successful cases compared to the currently used systemic chemotherapy 


\section{Liver Cancer}

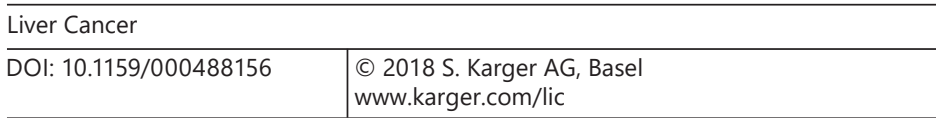

Moriguchi et al.: Hepatic Arterial Infusion Chemotherapy: A Potential Therapeutic Option for Hepatocellular Carcinoma with Portal Vein Tumor Thrombus

with sorafenib [2]. HAIC is not globally recommended as it has not been shown to be useful by randomized controlled trials; however, HAIC may be a useful treatment for advanced PVTT in HCC and is listed as a treatment option for HCC in the 2017 J-HCC clinical practice guideline. Lenvatinib, which has already demonstrated utility in a phase III trial, has relatively favorable response rates [3]. However, patients with tumor thrombus within the main trunk of the portal vein were excluded from this study, which is why HAIC may be a very important treatment option for advanced PVTT in HCC in clinical practice.

Furthermore, patients with advanced PVTT in HCC are often diagnosed with Child-Pugh class B or C, and in many cases, currently available systemic chemotherapy cannot be introduced. Although various systemic chemotherapeutic drugs are currently being developed, most target patients have Child-Pugh class A HCC. HAIC performed with the reservoir catheter system has been reported to be relatively safe, even in patients diagnosed with Child-Pugh class B [4], with relatively minor adverse events mainly including myelosuppression [1,2]; there are successfully treated cases in which hepatic reserve improves. Therefore, in ChildPugh class B or C HCC patients with advanced PVTT, if initial HAIC could successfully improve hepatic reserves to Child-Pugh class A under circumstances where the introduction of systemic chemotherapy is appropriate, for example in cases of extrahepatic spread, the therapeutic strategy of switching to systemic chemotherapy may be ideal. The Letter by Liu et al. [5] is exactly consistent with this strategy. In such cases, HAIC may be a rescue treatment or a bridging treatment for the introduction of systemic chemotherapy.

However, empirically, the efficacy of HAIC for cases that developed PVTT after repeated transcatheter arterial chemoembolization (TACE) was not good compared to cases not treated with TACE. Hence, for cases that developed PVTT after repeated sessions of TACE, HAIC may not be recommended. Future studies should examine the types of cases for which the advantage of HAIC can be maximized.

Regarding HAIC with the reservoir catheter system, there are several problems such as the skill involved in the placement of the reservoir catheter system and maintenance of the system for obtaining effective drug distribution. However, it seems a sufficiently feasible treatment method for sufficiently experienced physicians across institutions [6].

Recently, in a prospective phase III study (the SILIUS trial), Kudo et al. [7] reported that the primary endpoint was not met; however, on subanalysis, HAIC was effective for HCC with tumor thrombus involving the main trunk of the portal vein. It seems that HAIC could potentially be an important therapeutic option for advanced PVTT in HCC, from the utility and safety viewpoints, although a multicenter randomized controlled trial is needed for establishing HAIC as a treatment option.

\section{References}

1 Obi S, Sato S, Kawai T: Current status of hepatic arterial infusion chemotherapy. Liver Cancer 2015;4:188-199.

2 Moriguchi M, Aramaki T, Nishiofuku H, Sato R, Asakura K, Yamaguchi K, Tanaka T, Endo M, Itoh Y: Sorafenib versus hepatic arterial infusion chemotherapy as initial treatment for hepatocellular carcinoma with advanced portal vein tumor thrombosis. Liver Cancer. 2017;6:275-286.

-3 Kudo M, Finn RS, Qin S, Han KH, Ikeda K, Piscaglia F, Baron A, Park, JW, Han G, Jassem J, Blanc JF, Vogel A, Komov D, Evans TRJ, Lopez C, Dutcus C, Guo M, Saito K, Kraljevic S, Tamai T, Ren M, Cheng AL: Lenvatinib versus sorafenib in first-line treatment of patients with unresectable hepatocellular carcinoma: a randomised phase 3 non-inferiority trial. Lancet 2018;391:1163-1173.

4 Terashima T, Yamashita T, Arai K, Kawaguchi K, Kitamura K, Yamashita T, Sakai Y, Mizukoshi E, Honda M, Kaneko S: Response to chemotherapy improves hepatic reserve for patients with hepatocellular carcinoma and Child-Pugh B cirrhosis. Cancer Sci 2016;107:1263-1269.

5 Liu TH, Hsu CH, Shao YY: Successful hepatic arterial infusion of chemotherapy in a patient with advanced hepatocellular carcinoma and impending liver failure. Liver Cancer 2018, in press.

6 Arai Y, Ohtsu A, Sato Y, Aramaki T, Kato K, Hamada M, Muro K, Yamada Y, Inaba Y, Shimada Y, Boku N, Takeuchi Y, Morita S, Satake M: Phase I/II study of radiologic hepatic arterial infusion of fluorouracil plus systemic irinotecan for unresectable hepatic metastases from colorectal cancer: Japan Clinical Oncology Group Trial 0208-DI. J Vasc Interv Radiol 2012;23:1261-1267.

-7 Kudo M, Ueshima K, Yokosuka O, Ogasawara O, Obi S, Izumi N, Aikata H, Nagano H, Hatano E, Sasaki Y, Hino K, Kumada T, Yamamoto K, Imai Y, Iwadou S, Ogawa C, Okusaka T, Kanai F, Akazawa K, Yoshimura K, Johnson P, Arai Y; SILIUS study group: Randomised, open label, multicentre phase III trial comparing sorafenib plus low-dose cisplatin/fluorouracil hepatic arterial infusion chemotherapy with sorafenib alone in patients with advanced hepatocellular carcinoma: SILIUS Trial. Lancet Gastroenterol Hepatol 2018, in press. 